\title{
Extreme lengths in Brownian and Bessel excursions
}

\author{
YUEYUN HU and ZHAN SHI* \\ Université Paris VI, 4 place Jussieu, F-75252 Paris Cedex 05, France
}

We establish some strong limit theorems for the longest excursion lengths of a Bessel process of dimension $d \in(0,2)$. In the special case $d=1$, we recover and improve some well-known results for Wiener processes, and solve an open problem raised. The proof relies on exact distributions evaluated by Pitman and Yor and on a careful analysis of the Bessel sample paths.

Keywords: Bessel process; Brownian motion; excursion length; Lévy’s class

\section{Introduction}

Let $\{R(t) ; t \geqslant 0\}$ be a Bessel process of dimension $0<d<2$, starting from 0 (in the particular case $d=1, R$ becomes a reflecting Wiener process). For notational convenience, we write throughout the paper $v \equiv v(d)=(d-2) / 2$, which in Bessel language stands for the 'index' of $R$ (cf. Revuz and Yor 1994, Chapter XI). For any $t>0$, define $\Lambda^{-}(t)=$ $\sup \{s \leqslant t: R(s)=0\}$ and $\Lambda^{+}(t)=\inf \{s \geqslant t: R(s)=0\}$, which represent respectively the left and right extremities of the excursion interval straddling $t$. We are interested in

$$
V_{1}(t) \geqslant V_{2}(t) \geqslant \ldots \geqslant V_{n}(t) \geqslant \cdots
$$

the ordered excursion lengths of $R$ over $(0, t)$, the last zero-free interval $\left(\Lambda^{-}(t), t\right)$ being considered as an (incomplete) excursion interval. Discussions on excursion intervals excluding $\left(\Lambda^{-}(t), t\right)$ are postponed to Section 5 .

A remarkable development (Pitman and Yor 1992; 1997) in the study of ordered excursion lengths is that for any fixed positive numbers $r, s$ and $t$ and any integer $k \geqslant 1$,

$$
\begin{aligned}
& \left(\frac{V_{1}(t)}{t}, \frac{V_{2}(t)}{t}, \cdots\right) \stackrel{(d)}{=}\left(\frac{V_{1}(\tau(s))}{\tau(s)}, \frac{V_{2}(\tau(s))}{\tau(s)}, \cdots\right), \\
& \left(\frac{V_{1}(t)}{t}, \frac{V_{2}(t)}{t}, \cdots\right) \stackrel{(d)}{=}\left(\frac{V_{1}\left(H_{k}(r)\right)}{H_{k}(r)}, \frac{V_{2}\left(H_{k}(r)\right)}{H_{k}(r)}, \cdots\right),
\end{aligned}
$$

where ' $\stackrel{(d)}{=}$, stands for identity in distribution, $\tau$ is the right-continuous inverse process of the local time (in the sense of diffusion) of $R$ at 0 , and $H_{k}(r)=\inf \left\{t>0: V_{k}(t)=r\right\}$ is the first

\footnotetext{
*To whom correspondence should be addressed. e-mail: shi@ccr.jussieu.fr
} 
hitting time of $r$ by $V_{k}$. These identities in law, which bear similarities to Paul Lévy's celebrated arcsine laws, confirm random scaling properties for 'nice' clocks - a feature we shall exploit later. On the other hand, since $\tau$ is a stable subordinator of index $|v|$ (i.e. a nondecreasing $|v|$-stable process with independent increments; cf. Molchanov and Ostrovski 1969), the study in distribution of the $V_{k}$ is closely related to that of ranked jumps of stable subordinators. The latter has been the subject of considerable interest in the literature, with various motivations; see the references in Perman et al. (1992). Let us also mention recent applications of ordered Brownian excursion lengths in financial mathematics (Chesney et al. 1997).

Following Csáki et al. (1985), this paper is concerned with sample path properties of the longest lengths of the excursions.

Let us first consider $t \mapsto \sum_{j=1}^{k} V_{j}(t)$, the sum of the $k$ longest excursion lengths. Theorems 1.1 and 1.2 below characterize respectively the upper and lower functions of this process. Throughout the paper, 'i.o.' stands for 'infinitely often' as the appropriate index tends to $\infty$.

Theorem 1.1. Let $k \geqslant 1$ and let $\phi>0$ be a non-decreasing function. Then

$$
\mathbb{P}\left[\sum_{j=1}^{k} V_{j}(t)>t\left(1-\frac{1}{\phi(t)}\right) ; \text { i.o. }\right]=\left\{\begin{array} { l } 
{ 0 } \\
{ 1 }
\end{array} \Leftrightarrow \int ^ { \infty } \frac { \mathrm { d } t } { t ( \phi ( t ) ) ^ { | v | } } \left\{\begin{array}{l}
<\infty \\
=\infty
\end{array} .\right.\right.
$$

Theorem 1.2. For any fixed $k \geqslant 1$ and non-decreasing function $\psi>0$,

$$
\mathbb{P}\left[\sum_{j=1}^{k} V_{j}(t)<\frac{t}{\psi(t)} ; \text { i.o. }\right]=\left\{\begin{array} { l } 
{ 0 } \\
{ 1 }
\end{array} \Leftrightarrow \int ^ { \infty } \frac { \mathrm { d } t } { t } \psi ( t ) \operatorname { e x p } ( - k \gamma \psi ( t ) ) \left\{\begin{array}{l}
<\infty \\
=\infty
\end{array},\right.\right.
$$

where $\gamma \equiv \gamma(d)$ is the unique positive number such that

$$
\sum_{n=1}^{\infty} \frac{\gamma^{n}}{n !(n-|v|)}=\frac{1}{|v|}
$$

Remarks. (i) Taking $d=k=1$ in Theorems 1.1 and 1.2, we recover respectively the ChungErdös (1952) and Csáki-Erdös-Révész (1985) tests for the longest excursion of a Wiener process. For $d=1$ and arbitrary $k$, Theorem 1.2 improves the following iterated logarithm law established in Csáki et al. (1985):

$$
\liminf _{t \rightarrow \infty} \frac{\log \log t}{t} \sum_{j=1}^{k} V_{j}(t)=k \gamma \quad \text { a.s. }
$$

The somewhat intricate constant $\gamma$ appears naturally in Section 3.

(ii) Clearly Theorems 1.1 and 1.2 (as well as the forthcoming Theorems 1.3, 1.4 and 5.1) hold for all processes having the same zeros of a $d$-dimensional Bessel process. 
In order to obtain a more complete description of the excursion lengths, Csáki et al. (1985) raise the problem of characterizing the set of those non-decreasing functions $\phi$ for which

$$
\mathbb{P}\left[V_{2}(t) \geqslant \frac{t}{2}\left(1-\frac{1}{\phi(t)}\right) ; \text { i.o. }\right]=1 \text {. }
$$

We solve this problem for all the $V_{k}$.

Theorem 1.3. If $k \geqslant 2$ and if $\phi>0$ is non-decreasing, we have

$$
\mathbb{P}\left[V_{k}(t) \geqslant \frac{t}{k}\left(1-\frac{1}{\phi(t)}\right) ; \text { i.o. }\right]=\left\{\begin{array} { l } 
{ 0 } \\
{ 1 }
\end{array} \Leftrightarrow \int ^ { \infty } \frac { \mathrm { d } t } { t ( \phi ( t ) ) ^ { ( 1 + | v | ) k - 2 } } \left\{\begin{array}{c}
<\infty \\
=\infty
\end{array} .\right.\right.
$$

Finally, we complete the image of the limiting behaviours of the $V_{k}$ with an integral test which characterizes the lower functions.

Theorem 1.4. Assume $k \geqslant 2$ and $\psi>0$ non-decreasing. Then

$$
\mathbb{P}\left[V_{k}(t)<\frac{t}{\psi(t)} ; \text { i.o. }\right]=\left\{\begin{array} { l } 
{ 0 } \\
{ 1 }
\end{array} \Leftrightarrow \int ^ { \infty } \frac { \mathrm { d } t } { t ( \psi ( t ) ) ^ { | v | } } \left\{\begin{array}{c}
<\infty \\
=\infty
\end{array} .\right.\right.
$$

Remark. Despite the resemblance, Theorem 1.3 is of a different nature than the other theorems presented above, and its proof needs more care.

The rest of the paper is organized as follows. Theorem 1.3 is proved in Section 2 . Section 3 is devoted to the study of the upper and lower tails of $\sum_{j=1}^{k} V_{j}(t)$ and $V_{k}(t)$. The proofs of Theorems 1.1, 1.2 and 1.4 are provided in Section 4. The situation of completed excursion lengths is discussed in Section 5.

\section{Proof of Theorem 1.3}

Unless stated otherwise, $k \geqslant 2$ denotes a fixed integer in this section. The key ingredient in the proof of Theorem 1.3 is the following 'minimal inequality'. Recall that $H_{k}$ is the inverse process of $V_{k}$.

Lemma 2.1. There exists a constant $C \geqslant 1$ depending only on $k$ and $d$ such that for any $0<x<1 / k$

$$
C^{-1} x^{(|v|+1) k-2} \leqslant \mathbb{P}\left[\inf _{H_{k}(1) \leqslant t \leqslant H_{k}(2)}\left(\frac{1}{k}-\frac{V_{k}(t)}{t}\right)<x\right] \leqslant C x^{(|v|+1) k-2} .
$$

Before we continue, some comments on notation are necessary. First, in the rest of the paper, $C \geqslant 1$ denotes a finite constant. Its value, which may change from line to line, depends only on $k$ and on the underlying dimension $d$. Second, $a(x) \sim b(x)\left(x \rightarrow x_{0}\right)$ means 
$\lim _{x \rightarrow x_{0}} a(x) / b(x)=1 . \quad$ Third, $\quad a(x) \asymp b(x) \quad\left(x \rightarrow x_{0}\right) \quad$ means $\quad 0<\liminf _{x \rightarrow x_{0}} a(x) / b(x) \leqslant$ $\lim \sup _{x \rightarrow x_{0}} a(x) / b(x)<\infty$.

The proof of Lemma 2.1 relies on some exact distributions evaluated by Pitman and Yor (1997, Propositions 7 and 10), stated as follows.

Fact 2.2 (Pitman and Yor 1997). Let $\left\{V_{n}(1)\right\}_{n \geqslant 1}$ be as in (1.1). Then

$$
\left\{\frac{V_{n+1}(1)}{V_{n}(1)}\right\}_{n \geqslant 1}
$$

is a sequence of independent variables it such that $V_{n+1}(1) / V_{n}(1)$ has the $\operatorname{Beta}(n|v|, 1)$

distribution.

Furthermore, defining for $n \geqslant 1\left(\sum_{1}^{0} \equiv 0\right)$

$$
\Xi_{n}=\sum_{j=n+1}^{\infty} \frac{V_{j}(1)}{V_{n}(1)} \text { and } \mathrm{Y}_{n}=\sum_{j=1}^{n-1}\left(\frac{V_{j}(1)}{V_{n}(1)}-1\right)
$$

we have

$$
\begin{aligned}
& \mathbb{E} \exp \left(-\theta \Xi_{n}\right)=\left(1+|v| \int_{0}^{1} z^{-|v|-1}\left(1-\mathrm{e}^{-\theta z}\right) \mathrm{d} z\right)^{-n}, \\
& \mathbb{E} \exp \left(-\theta \mathrm{Y}_{n}\right)=\left(|v| \int_{0}^{\infty}(1+z)^{-|v|-1} \mathrm{e}^{-\theta z} \mathrm{~d} z\right)^{n-1}, \quad \theta \geqslant 0 .
\end{aligned}
$$

Remarks. (i) Elementary computations using (2.3a) and (2.4a) yield, for each fixed $n \geqslant 1$,

$$
\begin{aligned}
& \mathbb{E} \exp \left(-\theta \Xi_{n}\right) \sim(\Gamma(1-|v|))^{-n} \theta^{-n|v|}, \\
& \mathbb{E} \exp \left(-\theta \mathrm{Y}_{n}\right) \sim|v|^{n-1} \theta^{-(n-1)}, \quad \theta \rightarrow \infty
\end{aligned}
$$

(ii) By definition and (2.2), it immediately follows that

$$
\begin{aligned}
\frac{1}{V_{n}(1)} & =n+\mathrm{Y}_{n}+\Xi_{n}, \quad n \geqslant 1, \\
\frac{1}{V_{n}(1)}-1 & =\sum_{j=1}^{n-1} \frac{V_{j}(1)}{V_{n}(1)}+\Xi_{n}, \quad n \geqslant 1, \\
\frac{1}{V_{n}(1)}-1 & =\frac{V_{n-1}(1)}{V_{n}(1)}\left(n-1+\mathrm{Y}_{n-1}\right)+\Xi_{n}, \quad n \geqslant 2 .
\end{aligned}
$$

Proof of Lemma 2.1. Of course we only have to treat the case when $x$ is in the (positive) neighbourhood of 0 . For notational simplicity (i.e. by an abuse of notation!), we write $H_{k}=H_{k}(1)$ and $V_{k}=V_{k}(1)$ within the proof. Thus, with probability one,

$$
H_{k}-\Lambda^{-}\left(H_{k}\right)=1 .
$$


Define

$$
\begin{aligned}
X_{k} & =\inf _{H_{k}(1) \leqslant t \leqslant H_{k}(2)}\left(\frac{1}{k}-\frac{V_{k}(t)}{t}\right), \\
E & =\left\{1<V_{k-1}\left(H_{k}\right)<2\right\}, \\
F & =\left\{\Lambda^{+}\left(H_{k}\right)-\Lambda^{-}\left(H_{k}\right) \leqslant V_{k-1}\left(H_{k}\right)\right\} .
\end{aligned}
$$

Accordingly, the probability term in (2.1) can be written as

$$
\begin{aligned}
\mathbb{P}\left(X_{k}<x\right) & =\mathbb{P}\left(X_{k}<x ; E\right)+\mathbb{P}\left(X_{k}<x ; E^{\mathrm{c}}\right) \\
& \equiv \mathrm{I}+\mathrm{II},
\end{aligned}
$$

with obvious notation. We now evaluate I and II. Observe that if $\omega \in E$, then $H_{k}(2)$ is strictly larger than $\Lambda^{+}\left(H_{k}\right)$, and several lines of elementary calculation show that $\sup _{\Lambda^{+}\left(H_{k}\right) \leqslant t \leqslant H_{k}(2)} V_{k}(t) / t$ is either realized at $t=\Lambda^{+}\left(H_{k}\right)$ or smaller than $1 /\left(k+\frac{1}{2}\right)$. Thus in this situation,

$$
\left\{X_{k}<x\right\}=\left\{\inf _{H_{k} \leqslant t \leqslant \Lambda^{+}\left(H_{k}\right)}\left(\frac{1}{k}-\frac{V_{k}(t)}{t}\right)<x\right\} .
$$

We distinguish two subcases. First, let $\omega \in E \cap F$. We have $V_{k}(t)=t-\Lambda^{-}\left(H_{k}\right)$ for $t \in\left[H_{k}, \Lambda^{+}\left(H_{k}\right)\right]$, which means that the supremum of $V_{k}(t) / t$ over $\left[H_{k}, \Lambda^{+}\left(H_{k}\right)\right]$ is reached at $t=\Lambda^{+}\left(H_{k}\right)$, or equivalently that $X_{k}=\Lambda^{-}\left(H_{k}\right) / \Lambda^{+}\left(H_{k}\right)-(k-1) / k$. Accordingly,

$$
\left(\left\{X_{k}<x\right\} \cap E \cap F\right) \subset\left(E \cap F \cap\left\{\Lambda^{-}\left(H_{k}\right) \leqslant \frac{k-1+k x}{1-k x} V_{k-1}\left(H_{k}\right)\right\}\right) .
$$

In the second subcase, $\omega \in E \cap F^{\mathrm{c}}$, it is easily seen that the supremum of $V_{k}(t) / t$ over $\left[H_{k}, \Lambda^{+}\left(H_{k}\right)\right]$ is realized at $t=\Lambda^{-}\left(H_{k}\right)+V_{k-1}\left(H_{k}\right)$. Thus $X_{k}=1 / k-V_{k-1}\left(H_{k}\right) /$ $\left(\Lambda^{-}\left(H_{k}\right)+V_{k-1}\left(H_{k}\right)\right)$, which yields

$$
\left(\left\{X_{k}<x\right\} \cap E \cap F^{\mathfrak{c}}\right)=\left(E \cap F^{\mathrm{c}} \cap\left\{\Lambda^{-}\left(H_{k}\right) \leqslant \frac{k-1+k x}{1-k x} V_{k-1}\left(H_{k}\right)\right\}\right) .
$$

By (2.9) and (2.10), we obtain

$$
\begin{aligned}
\mathrm{I} & =\mathbb{P}\left(X_{k}<x ; E ; F\right)+\mathbb{P}\left(X_{k}<x ; E ; F^{\mathrm{c}}\right) \\
& \leqslant \mathbb{P}\left(E ; \Lambda^{-}\left(H_{k}\right) \leqslant \frac{k-1+k x}{1-k x} V_{k-1}\left(H_{k}\right)\right) \\
& \leqslant \mathbb{P}\left(1<V_{k-1}\left(H_{k}\right)<2 ; H_{k}-1 \leqslant\left(k-1+2 k^{2} x\right) V_{k-1}\left(H_{k}\right)\right),
\end{aligned}
$$


the last inequality being due to (2.8) and the trivial estimate $(k-1+k x) /(1-k x) \leqslant k-1+$ $2 k^{2} x$ (for small $x$ ). Since $V_{k}\left(H_{k}\right)=1$, by means of (1.3) and (2.7), we have, for any $z>0$,

$$
\begin{aligned}
\mathbb{P} & \left(1<V_{k-1}\left(H_{k}\right)<2 ; H_{k}-1 \leqslant(k-1+z) V_{k-1}\left(H_{k}\right)\right) \\
= & \mathbb{P}\left(1<\frac{V_{k-1}}{V_{k}}<2 ; \frac{1}{V_{k}}-1<(k-1+z) \frac{V_{k-1}}{V_{k}}\right) \\
= & \mathbb{P}\left(1<\frac{V_{k-1}}{V_{k}}<2 ; \mathrm{Y}_{k-1}+\frac{V_{k}}{V_{k-1}} \Xi_{k}<z\right) \\
& \leqslant \mathbb{P}\left(\mathrm{Y}_{k-1}+\frac{1}{2} \Xi_{k}<z\right) .
\end{aligned}
$$

Since $\mathrm{Y}_{k-1}$ and $\Xi_{k}$ are independent (cf. (2.2)), using (2.3b) and (2.4b), we have, for some unimportant (but computable) constant $C>0$,

$$
\mathbb{E} \exp \left(-\theta \mathrm{Y}_{k-1}-\frac{\theta}{2} \Xi_{k}\right) \sim C \theta^{-(1+|v|) k+2}, \quad \theta \rightarrow \infty
$$

It follows from a Tauberian theorem (cf. Feller 1971, p. 445) that

$$
\mathbb{P}\left(\mathrm{Y}_{k-1}+\frac{1}{2} \Xi_{k}<y\right) \asymp y^{(1+|v|) k-2}, \quad y \rightarrow 0 .
$$

Going back to (2.11) and (2.13), we obtain

$$
\mathrm{I} \leqslant C x^{(1+|v|) k-2} .
$$

To estimate II, consider the event $E^{\mathrm{c}}=\left\{V_{k-1}\left(H_{k}\right) \geqslant 2\right\}$. A key observation is that in order to realize $\left\{X_{k}<x\right\}$ ( $x$ being sufficiently small), we must have $H_{k}(2) \leqslant \Lambda^{+}\left(H_{k}\right)$. Moreover, the supremum of $t \mapsto V_{k}(t) / t$ over $\left[H_{k}(1), H_{k}(2)\right]$ is realized at $H_{k}(2)=H_{k}(1)+1$, and it is easily seen that $V_{1}\left(H_{k}\right) \leqslant 2+2 k^{2} x /(1-k x)$ (which is smaller than $2+3 k^{2} x$ ). Consequently,

$$
\begin{aligned}
\mathrm{II} & =\mathbb{P}\left(X_{k}<x ; E^{\mathrm{c}}\right) \\
& \leqslant \mathbb{P}\left(\frac{1}{k}-\frac{2}{H_{k}+1}<x ; 2 \leqslant V_{j}\left(H_{k}\right) \leqslant 2+3 k^{2} x, j=1, \ldots, k-1\right) \\
& =\mathbb{P}\left(\frac{1}{k}-\frac{2}{1+1 / V_{k}}<x ; 2 \leqslant \frac{V_{j}}{V_{k}} \leqslant 2+3 k^{2} x, j=1, \ldots, k-1\right),
\end{aligned}
$$

the last identity being due to (1.3). Using (2.6) and writing $V_{j} / V_{k}=\prod_{i=j}^{k-1}\left(V_{i} / V_{i+1}\right)$, we obtain

$\mathrm{II} \leqslant \mathbb{P}\left(\Xi_{k}<\frac{2}{1 / k-x}-2 k ; 2 \leqslant \frac{V_{k-1}}{V_{k}} \leqslant 2+3 k^{2} x ; 1 \leqslant \frac{V_{j}}{V_{j+1}} \leqslant 1+2 k^{2} x, j=1, \ldots, k-2\right)$. 
Since $\Xi_{k}$ and $\left(V_{j+1} / V_{j}\right)_{1 \leqslant j \leqslant k-1}$ are independent such that $V_{j+1} / V_{j}$ has $\operatorname{Beta}(j|v|, 1)$, law (cf. (2.2)), this implies

$$
\mathrm{II} \leqslant C x^{k-1} \mathbb{P}\left(\Xi_{k}<3 k^{2} x\right) .
$$

The lower tail of $\Xi_{k}$ is easily computed. Indeed, by $(2.3 \mathrm{~b})$ and the Tauberian theorem mentioned above,

$$
\mathbb{P}\left(\Xi_{k}<y\right) \asymp y^{k|v|}, \quad y \rightarrow 0 .
$$

Consequently,

$$
\mathrm{II} \leqslant C x^{(1+|v|) k-1} .
$$

Since $\mathbb{P}\left(X_{k}<x\right)=\mathrm{I}+\mathrm{II}$, combining (2.15) and (2.17) yields the second part of Lemma 2.1. It remains to verify its first part. Observe from (2.10) and (2.8) that

$$
\left(\left\{X_{k}<x\right\} \cap E \cap B\right)=(E \cap A \cap B),
$$

with

$$
\begin{aligned}
& A=\left\{\Lambda^{-}\left(H_{k}\right) \leqslant \frac{k-1+k x}{1-k x} V_{k-1}\left(H_{k}\right)\right\}, \\
& B=\left\{1<\frac{\Lambda^{+}\left(H_{k}\right)-H_{k}+1}{V_{k-1}\left(H_{k}\right)}<13\right\} .
\end{aligned}
$$

Let us now estimate $\mathbb{P}(E \cap A \cap B)$. First, the random variable $\left(\Lambda^{+}\left(H_{k}\right)-H_{k}\right) /\left(R\left(H_{k}\right)\right)^{2}$ is independent of $\mathscr{F}_{H_{k}}(\mathscr{F}$ being the completed natural filtration of $R)$, and has the same law as the first hitting time at 0 of a $d$-dimensional Bessel process starting from 1 . Thus

$$
\begin{aligned}
\mathbb{P}(E \cap A \cap B) & \geqslant \mathbb{P}\left(E ; A ; B ; 2<\frac{\Lambda^{+}\left(H_{k}\right)-H_{k}}{\left(\mathrm{R}\left(H_{k}\right)\right)^{2}}<3\right) \\
& \geqslant C^{-1} \mathbb{P}\left(E \cap A ; \frac{1}{4}<\frac{V_{k-1}\left(H_{k}\right)}{\left(R\left(H_{k}\right)\right)^{2}}<2\right),
\end{aligned}
$$

with $C^{-1}=\mathbb{P}\left(2<\left(\Lambda^{+}\left(H_{k}\right)-H_{k}\right) /\left(R\left(H_{k}\right)\right)^{2}<3\right)$. Since $R\left(H_{k}\right)$ is independent of $\mathscr{F} \Lambda^{-}\left(H_{k}\right)$ - this is pointed out in Lecture 6 of Yor (1995) for the Brownian case; the independence holds, however, for general Markov processes (cf. Jeulin 1980, Theorem 6.3) we have

$$
\mathbb{P}(E \cap A \cap B) \geqslant C^{-1} \mathbb{P}\left(1<R\left(H_{k}\right)<2\right) \mathbb{P}(E \cap A)=\hat{C}^{-1} \mathbb{P}(E \cap A) .
$$

By means of (2.12) and the independence of $\mathrm{Y}_{k-1}, \Xi_{k}$ and $V_{k-1} / V_{k}$ (cf. (2.2)), we obtain

$$
\begin{aligned}
\mathbb{P}(E \cap A) & \geqslant \mathbb{P}\left(\mathrm{Y}_{k-1}+\Xi_{k}<k x\right) \mathbb{P}\left(1<\frac{V_{k-1}}{V_{k}}<2\right) \\
& \geqslant C^{-1} \mathbb{P}\left(\mathrm{Y}_{k-1}+\frac{1}{2} \Xi_{k}<\frac{1}{2} k x\right),
\end{aligned}
$$


which, according to (2.18) and (2.14), implies

$$
\mathbb{P}\left(X_{k}<x ; E ; B\right) \geqslant C^{-1} x^{(1+|v|) k-2} .
$$

This is the desired first part of Lemma 2.1.

From now on, we shall properly write $V_{k}(1)$ for the variable, $V_{k}$ denoting exclusively the process $t \mapsto V_{k}(t)$.

Proof of Theorem 1.3. Let $\phi>0$ be non-decreasing such that $\int \infty(\mathrm{d} t / t)(\phi(t))^{-(1+|v|) k+2}<\infty$. Define $t_{n}=2^{n}$. By scaling and Lemma 2.1,

$$
\begin{aligned}
\mathbb{P}\left[\inf _{H_{k}\left(t_{n}\right) \leqslant t \leqslant H_{k}\left(t_{n+1}\right)}\left(\frac{1}{k}-\frac{V_{k}(t)}{t}\right)<\frac{1}{\phi\left(t_{n}\right)}\right] & =\mathbb{P}\left[\inf _{H_{k}(1) \leqslant t \leqslant H_{k}(2)}\left(\frac{1}{k}-\frac{V_{k}(t)}{t}\right)<\frac{1}{\phi\left(t_{n}\right)}\right] \\
& \leqslant C\left(\phi\left(t_{n}\right)\right)^{-(1+|v|) k+2},
\end{aligned}
$$

which is summable for $n$. According to the Borel-Cantelli lemma, when $n$ is sufficiently large and $t \in\left[H_{k}\left(t_{n}\right), H_{k}\left(t_{n+1}\right)\right]$, we have

$$
\frac{1}{k}-\frac{V_{k}(t)}{t} \geqslant \frac{1}{\phi\left(t_{n}\right)} \geqslant \frac{1}{\phi\left(V_{k}(t)\right)} \geqslant \frac{1}{\phi(t)},
$$

which yields the convergent half of Theorem 1.3. For its divergent half, we assume that $\int^{\infty}(\mathrm{d} t / t)(\phi(t))^{-(1+|v|) k+2}=\infty$ and let $t_{n}=2^{n}$. Define $\hat{\phi}(t)=\phi\left(t^{2}\right)$ and consider

$$
\begin{aligned}
& D_{n}=\left\{\inf _{H_{k}\left(t_{n}\right) \leqslant t \leqslant H_{k}\left(t_{n+1}\right)}\left(\frac{1}{k}-\frac{V_{k}(t)}{t}\right)<\frac{1}{\hat{\phi}\left(t_{n}\right)}\right\}, \\
& E_{n}=\left\{1<\frac{V_{k-1}\left(H_{k}\left(t_{n}\right)\right)}{t_{n}}<2\right\}, \\
& B_{n}=\left\{1<\frac{\Lambda^{+}\left(H_{k}\left(t_{n}\right)\right)-\Lambda^{-}\left(H_{k}\left(t_{n}\right)\right)}{V_{k-1}\left(H_{k}\left(t_{n}\right)\right)}<13\right\}, \\
& G_{n}=D_{n} \cap E_{n} \cap B_{n},
\end{aligned}
$$

for $n \geqslant n_{0}$. Recall that $t_{n+1}=2 t_{n}$ and $H_{k}\left(t_{n}\right)=\Lambda^{-}\left(H_{k}\left(t_{n}\right)\right)+t_{n}$; by scaling and (2.19), we obtain

$$
\mathbb{P}\left(G_{n}\right) \geqslant C^{-1}\left(\hat{\phi}\left(t_{n}\right)\right)^{-(1+|v|) k+2},
$$

which implies $\sum_{n} \mathbb{P}\left(G_{n}\right)=\infty$. Of course, we intend to apply the Borel-Cantelli lemma. To this end, observe that on $D_{n} \cap E_{n}$ (with sufficiently large $n$ ), the infimum expression in $D_{n}$ must be realized at some (random) time $t \in\left[H_{k}\left(t_{n}\right), \Lambda^{+}\left(H_{k}\left(t_{n}\right)\right)\right]$; furthermore, in this case the length of any excursion before $H_{k}\left(t_{n}\right)$ must be less than $3 t_{n}$. Accordingly, if 
$\omega \in D_{n} \cap E_{n} \cap B_{n}$, then any excursion before $\Lambda^{+}\left(H_{k}\left(t_{n}\right)\right)$ has length no longer than $26 t_{n}$, i.e.

$$
\begin{aligned}
G_{n}= & E_{n} \cap B_{n} \cap\left\{\inf _{H_{k}\left(t_{n}\right) \leqslant t \leqslant \Lambda^{+}\left(H_{k}\left(t_{n}\right)\right)}\left(\frac{1}{k}-\frac{V_{k}(t)}{t}\right)<\frac{1}{\hat{\phi}\left(t_{n}\right)}\right\} \\
& \cap\left\{V_{1}\left(\Lambda^{+}\left(H_{k}\left(t_{n}\right)\right)\right)<26 t_{n}\right\} .
\end{aligned}
$$

Consider now $j-6 \geqslant i \geqslant n_{0}$ (with a sufficiently large initial value $n_{0}$ ). Let $\{\hat{R}(t) \equiv$ $\left.R\left(t+\Lambda^{+}\left(H_{k}\left(t_{i}\right)\right)\right) ; t \geqslant 0\right\}$, which is a $d$-dimensional Bessel process starting from 0 , independent of $\mathscr{F}_{\Lambda^{+}\left(H_{k}\left(t_{i}\right)\right)}\left(\mathscr{F}\right.$ being the filtration of $R$ ). Define the corresponding $\left(\hat{V}_{k}(t)\right)$ and $\left(\hat{H}_{k}(t)\right)$ in an obvious way. Since $t_{j}>26 t_{i+1}$, we deduce from (2.21) that on the event $G_{i}, \quad H_{k}(s)=\hat{H}_{k}(s)+\Lambda^{+}\left(H_{k}\left(t_{i}\right)\right)$ for $t_{j} \leqslant s \leqslant t_{j+1}$ and $V_{k}(t)=\hat{V}_{k}\left(t-\Lambda^{+}\left(H_{k}\left(t_{i}\right)\right)\right)$ for $H_{k}\left(t_{j}\right) \leqslant t \leqslant H_{k}\left(t_{j+1}\right)$. The strong Markov property yields

$$
\begin{aligned}
\mathbb{P}\left(G_{i} \cap G_{j}\right) & \leqslant \mathbb{P}\left(G_{i} ; \inf _{\hat{H}_{k}\left(t_{j}\right) \leqslant u \leqslant \hat{H}_{k}\left(t_{j+1}\right)}\left(\frac{1}{k}-\frac{\hat{V}_{k}(u)}{u+\Lambda^{+}\left(H_{k}\left(t_{i}\right)\right)}\right)<\frac{1}{\hat{\phi}\left(t_{i}\right)}\right) \\
& \leqslant \mathbb{P}\left(G_{i} ; \inf _{\hat{H}_{k}\left(t_{j}\right) \leqslant u \leqslant \hat{H}_{k}\left(t_{j+1}\right)}\left(\frac{1}{k}-\frac{\hat{V}_{k}(u)}{u}\right)<\frac{1}{\hat{\phi}\left(t_{i}\right)}\right) \\
& =\mathbb{P}\left(G_{i}\right) \mathbb{P}\left(D_{j}\right) \\
& \leqslant C \mathbb{P}\left(G_{i}\right) \mathbb{P}\left(G_{j}\right),
\end{aligned}
$$

where in the last inequality we have used the scaling property, Lemma 2.1, as well as (2.20). Consequently,

$$
\liminf _{n \rightarrow \infty} \sum_{i=n_{0}}^{n} \sum_{j=n_{0}}^{n} \mathbb{P}\left(G_{i} \cap G_{j}\right) /\left(\sum_{i=n_{0}}^{n} \mathbb{P}\left(G_{i}\right)\right)^{2} \leqslant C .
$$

According to Kochen and Stone's (1964) Borel-Cantelli lemma, We have $\mathbb{P}\left(G_{n}\right.$; i.o.) $\geqslant 1 / C$. Now observe that with probability one, we have $V_{k}(t) \geqslant 2 t^{1 / 2}$ for sufficiently large $t$ (this can easily be verified by means of the convergent part of the Borel-Cantelli lemma; actually more is true for this kind of estimate, cf. Theorem 1.4). Hence

$$
\mathbb{P}\left[V_{k}(t) \geqslant \frac{t}{k}\left(1-\frac{1}{\phi(t)}\right) ; \text { i.o. }\right]>0 .
$$

This yields the divergent half once we prove the following lemma.

Lemma 2.3. Let $k \geqslant 1$, and let $f>0$ be a measurable function such that $f(t) \rightarrow \infty$ (as $t \rightarrow \infty)$. The event

$$
\left\{V_{k}(t) \geqslant f(t) ; \text { i.o. }\right\}
$$

has probability zero or one. 
Proof. Fix $s>0$. Consider the new Bessel process $\left\{R^{(s)}(t) \equiv R\left(t+\Lambda^{+}(s)\right) ; t \geqslant 0\right\}$. Denote by $V_{1}^{(s)} \geqslant V_{2}^{(s)} \geqslant \ldots$ the associated ranked excursion lengths processes. Clearly,

$$
\left\{V_{k}(t) \geqslant f(t) \text {; i.o. }\right\}=\left\{V_{k}^{(s)}(t) \geqslant f\left(t+\Lambda^{+}(s)\right) \text {; i.o. }\right\} .
$$

Since $\Lambda^{+}(s)$ and the process $R^{(s)}$ (hence $V_{k}^{(s)}$ ) are measurable with respect to $\mathscr{G}_{s} \equiv$ $\sigma\{R(u) ; u \geqslant s\}$, so is the event on the right-hand-side of (2.22). Consequently, $\left\{V_{k}(t) \geqslant\right.$ $f(t)$ : i.o. $\}$ is $\cap_{r \geqslant s} \mathscr{G}_{r}$-measurable, for all $s>0$, which means that it is a trivial event (this is easily seen, for example, by virtue of Bessel time inversion and Blumenthal's 0-1 law).

\section{Tails}

The following results give useful estimates of the tails of $V_{k}(1)$ and of $\sum_{j=1}^{k} V_{j}(1)$.

Theorem 3.1. As $x \rightarrow 0$,

$$
\begin{aligned}
\mathbb{P}\left(\sum_{j=1}^{k} V_{j}(1)<x\right) & \asymp \exp \left(-\frac{k \gamma}{x}\right), \quad k \geqslant 1, \\
\mathbb{P}\left(\sum_{j=1}^{k} V_{j}(1)>1-x\right) & \asymp x^{|v|}, \quad k \geqslant 1, \\
\mathbb{P}\left(V_{k}(1)<x\right) & \asymp x^{|v|}, \quad k \geqslant 2,
\end{aligned}
$$

where $\gamma$ is defined in Theorem 1.2.

Before proving Theorem 3.1, we state a Tauberian theorem which may be of independent interest.

Theorem 3.2. Let $a, b$ and $c$ be positive constants, and $A$ a non-increasing function with $\lim _{s \rightarrow \infty} A(s)$ such that

$$
F(z) \equiv-\int_{0}^{\infty} \mathrm{e}^{z s} \mathrm{~d} A(s)
$$

converges for $\operatorname{Re}(z)<a$. Define $G(s) \equiv F(a-s) /(a-s)-c / s^{b}$ for $0<\operatorname{Re}(s)<a$. If for any fixed $T>0$,

$$
\eta(x, T) \equiv x^{b-1} \int_{-T}^{T}|G(2 x+\mathrm{i} y)-G(x+\mathrm{i} y)| \mathrm{d} y=o(1), \quad x \rightarrow 0^{+},
$$

then

$$
A(t)=\left(\frac{c}{\Gamma(b)}+O(\rho(t))\right) \mathrm{e}^{-a t} t^{b-1}, \quad t \rightarrow \infty
$$


with $\rho(t) \equiv \inf _{T \geqslant 1}\left(T^{-1}+\eta\left(t^{-1}, T\right)+(t T)^{-b}\right)$. The implicit constants in $O(\rho(t))$ above depend only on $a, b$ and $c$.

Remark. In most probabilistic applications, $A(t)$ is the tail distribution of some random variable, say $X$, and $F(z)$ the corresponding exponential moment which explodes at $\operatorname{Re}(z)=a$. If we know the rate of explosion of $F(z)$ at $\operatorname{Re}(z)=a$ (and under the regularity condition on $\eta(x, T)$, of course), Theorem 3.2 provides useful information about the upper tail behaviour of $X$. Note that no additional information is required about $G(s)$ when $\operatorname{Re}(s) \rightarrow a$ (which corresponds to $\operatorname{Re}(z) \rightarrow 0$ for the exponential moment $F(z)$ ). The 'critical line' for $G(s)$ is $\operatorname{Re}(s)=0$ (the value of $b$ contains the main information about the explosion rate), not $\operatorname{Re}(s)=a$.

Proof of Theorem 3.2. Suppose without loss of generality that $A\left(0^{+}\right)=1$. Define $A(t) \equiv 1$ for $t \leqslant 0$ and $g_{\sigma}(t) \equiv\left(1-\mathrm{e}^{-\sigma t}\right) \mathrm{e}^{(a-\sigma) t} A(t), 0 \leqslant \sigma<a / 2$. The proof is along the lines presented in Tenenbaum (1995, p. 234, Theorem 11) who treats the case when $A$ is nondecreasing. The details are omitted. We only mention the key estimate: there exists a constant $K>0$ depending only on $a, b$ and $c$, such that for all $T \geqslant 1,0 \leqslant y \leqslant 1 / T, 0 \leqslant \sigma<a / 2$,

$$
\sup _{-\infty<x<\infty}\left(g_{\sigma}(x+y)-g_{\sigma}(x)\right) \leqslant K\left\|g_{\sigma}\right\|_{\infty} y,
$$

(' $\|\cdot\|_{\infty}$ ' denoting the $L_{\infty}$-norm). This is verified via several lines of careful though elementary calculation.

Proof of Theorem 3.1. By (2.3a) and analytic continuation, we have, for $\theta<\gamma$,

$$
\begin{aligned}
\mathbb{E} \exp \left(\theta \Xi_{k}\right) & =\left(1-|v| \int_{0}^{1} z^{-|v|-1}\left(\mathrm{e}^{\theta z}-1\right) \mathrm{d} z\right)^{-k} \\
& =\left(1-|v| \sum_{n=1}^{\infty} \frac{\theta^{n}}{n !(n-|v|)}\right)^{-k} .
\end{aligned}
$$

Applying Theorem 3.2 to $A(t)=\mathbb{P}\left(\Xi_{k}>t\right), a=\gamma, b=k$ and $c=\gamma^{-1}\left(|v| \int_{0}^{1} x^{-|v|} \mathrm{e}^{-\gamma x} \mathrm{~d} x\right)^{-k}$, this yields

$$
\mathbb{P}\left(\Xi_{k}>y\right) \sim \frac{c}{\Gamma(k)} y^{k-1} \exp (-\gamma y), \quad y \rightarrow \infty
$$

On the other hand, using (2.4b) gives

$$
\mathbb{E}\left[\left(k+\mathrm{Y}_{k}\right)^{k-1} \exp \left(-\lambda \mathrm{Y}_{k}\right)\right] \asymp \lambda^{-(k-1)}, \quad \lambda \rightarrow \infty \text {. }
$$

By (2.5) and (2.6),

$$
\frac{1}{\sum_{j=1}^{k} V_{j}(1)}=1+\frac{\Xi_{k}}{k+\mathrm{Y}_{k}}
$$


Since $\Xi_{k}$ and $\mathrm{Y}_{k}$ are independent, (3.4)-(3.6) together imply

$$
\begin{aligned}
\mathbb{P}\left(\frac{1}{\sum_{1}^{k} V_{j}(1)}>z+1\right) & \sim \frac{c}{\Gamma(k)} z^{k-1} \mathbb{E}\left[\left(k+\mathrm{Y}_{k}\right)^{k-1} \exp \left(-\gamma z\left(k+\mathrm{Y}_{k}\right)\right)\right] \\
& \asymp \exp (-k \gamma z), \quad z \rightarrow \infty,
\end{aligned}
$$

which yields (3.1). Similarly, combining (2.4b) with (2.16) and (3.6) implies (3.2), and (3.3) is a straightforward consequence of (2.5), (2.3b) and (2.4b).

\section{Proofs of Theorems 1.1, 1.2 and 1.4}

The convergent part of these theorems follows from the tail estimations in Theorem 3.1, the scaling property, and a standard argument combining the easy half of the Borel-Cantelli lemma and monotonicity.

Proof of the convergent part of Theorems 1.1, 1.2 and 1.4. Let $k \geqslant 1$ and let $\phi$ be nondecreasing such that $\int^{\infty}(\mathrm{d} t / t) \phi(t)^{-|v|}<\infty$. Define $t_{n}=2^{n}$ for $n \geqslant n_{0}$. Since $\sum_{j=1}^{\infty} V_{j}\left(t_{n}\right)$ $=1$ and $t_{n+1}=2 t_{n}$, by scaling and (3.2), we have

$$
\mathbb{P}\left(\sum_{j=k+1}^{\infty} V_{j}\left(t_{n}\right)<\frac{t_{n+1}}{\phi\left(t_{n}\right)}\right)=\mathbb{P}\left(\sum_{j=1}^{k} V_{j}(1)>1-\frac{2}{\phi\left(t_{n}\right)}\right) \leqslant C\left(\phi\left(t_{n}\right)\right)^{|v|},
$$

and the sum over $n$ of the latter quantity is finite. Thus by the Borel-Cantelli lemma, almost surely for sufficiently large $n$, we have $\sum_{j=k+1}^{\infty} V_{j}\left(t_{n}\right) \geqslant t_{n+1} / \phi\left(t_{n}\right)$. Let $t \in\left[t_{n}, t_{n+1}\right]$. Then

$$
\sum_{j=1}^{k} V_{j}(t)=t-\sum_{j=k+1}^{\infty} V_{j}(t) \leqslant t-\sum_{j=k+1}^{\infty} V_{j}\left(t_{n}\right) \leqslant t-\frac{t_{n+1}}{\phi\left(t_{n}\right)} \leqslant t\left(1-\frac{1}{\phi(t)}\right),
$$

which implies the convergent half of Theorem 1.1. That of Theorems 1.2 and 1.4 can be proved along the same lines.

Proof of the divergent half of Theorem 1.2. In view of the obvious relation $\sum_{j=1}^{k} V_{j}(t) \leqslant$ $k V_{1}(t)$, it suffices to treat the case $k=1$. Let $\int{ }^{\infty}(\mathrm{d} t / t) \psi(t) \exp (-\gamma \psi(t))=\infty$. As usual for this kind of exponential integral test, we can limit ourselves to the study of a 'critical case':

$$
\frac{1}{2 \gamma} \log \log t \leqslant \psi(t) \leqslant \frac{2}{\gamma} \log \log t
$$

(cf. Erdös 1942). Define $t_{i}=\exp (i / \log i)$ and $A_{i}=\left\{V_{1}\left(t_{i}\right)<t_{i} / \psi\left(t_{i}\right)\right\}$ for $i \geqslant n_{0}\left(n_{0}\right.$ being sufficiently large). By scaling and taking $k=1$ in (3.1),

$$
\mathbb{P}\left(A_{i}\right) \geqslant C^{-1} \exp \left(-\gamma \psi\left(t_{i}\right)\right) \text {, }
$$


which implies $\sum_{i} \mathbb{P}\left(A_{i}\right)=\infty$. Let $j>i \geqslant n_{0}$. By the Markov property, we have

$$
\begin{aligned}
\mathbb{P}\left(A_{i} \cap A_{j}\right) & \leqslant \mathbb{P}\left(A_{i} ; \text { the longest length of excursions over }\left(t_{i}, t_{j}\right)<\frac{t_{j}}{\psi\left(t_{j}\right)}\right) \\
& =\mathbb{E}\left[\int_{A_{i}} \mathbb{P}_{R\left(t_{i}\right)}\left(V_{1}\left(t_{j}-t_{i}\right)<\frac{t_{j}}{\psi\left(t_{j}\right)}\right)\right],
\end{aligned}
$$

where $\mathbb{P}_{a}$ denotes the probability under which the Bessel process starts from $a$ (thus $\left.\mathbb{P}=\mathbb{P}_{0}\right)$. By (3.1), $\mathbb{P}\left(V_{1}(u)<y\right) \leqslant C \exp (-\gamma u / y)$ (for any $u$ and $y$ ). Therefore, if $T(0)=\inf \{t>0: R(t)=0\}$ denotes the first hitting time at 0 by $R$, then for any positive numbers $a$ and $t>y$,

$$
\begin{aligned}
\mathbb{P}_{a}\left(V_{1}(t)<y\right) & =\int_{0}^{y} \mathbb{P}_{a}(T(0) \in \mathrm{d} s) \mathbb{P}\left(V_{1}(t-s)<y\right) \\
& \leqslant C \mathrm{e}^{\gamma} \mathrm{e}^{-\gamma t / y} \int_{0}^{y} \mathbb{P}_{a}(T(0) \in \mathrm{d} s) \\
& \leqslant C \mathrm{e}^{\gamma} \mathrm{e}^{-\gamma t / y}
\end{aligned}
$$

Going back to (4.3), we obtain

$$
\mathbb{P}\left(A_{i} \cap A_{j}\right) \leqslant C \mathbb{P}\left(A_{i}\right) \exp \left(-\gamma\left(1-\frac{t_{i}}{t_{j}}\right) \psi\left(t_{j}\right)\right) .
$$

From here, we use an argument due to Erdös (1942). Let us distinguish two possible situations. First, assume $j<i+(\log i)^{2}$. In this case, using (4.1) and the definition of the sequence $\left(t_{n}\right)$, we easily arrive at the estimate $\left(1-t_{i} / t_{j}\right) \psi\left(t_{j}\right) \geqslant \min (j-i, \log i) / C$, which according to (4.4) yields

$$
\begin{aligned}
\sum_{n_{0} \leqslant i \leqslant j \leqslant n ; j<i+(\log i)^{2}} \sum_{i} \mathbb{P}\left(A_{i} \cap A_{j}\right) & \leqslant C \sum_{i=n_{0}}^{n} \mathbb{P}\left(A_{i}\right) \sum_{j=i}^{\infty} \mathrm{e}^{-(j-i) / C}+C \sum_{i=n_{0}}^{n} \mathbb{P}\left(A_{i}\right) \sum_{j=i}^{i+(\log i)^{2}} i^{-1 / C} \\
& \leqslant \hat{C} \sum_{i=n_{0}}^{n} \mathbb{P}\left(A_{i}\right)
\end{aligned}
$$

for some constant $\hat{C}$ depending only on $d$. If on the other hand $j \geqslant i+(\log i)^{2}$, then by (4.1), $\left(t_{i} / t_{j}\right) \psi\left(t_{j}\right)$ is bounded (above). Therefore we have, by (4.4) and (4.2), $\mathbb{P}\left(A_{i} \cap A_{j}\right) \leqslant$ $C \mathbb{P}\left(A_{i}\right) \mathbb{P}\left(A_{j}\right)$, which implies

$$
\sum_{n_{0} \leqslant i \leqslant j \leqslant n ; j \geqslant i+(\log i)^{2}} \mathbb{P}\left(A_{i} \cap A_{j}\right) \leqslant C\left(\sum_{i=n_{0}}^{n} \mathbb{P}\left(A_{i}\right)\right)^{2} .
$$

Since $\sum \mathbb{P}\left(A_{i}\right)=\infty$, combining (4.5) and (4.6) gives

$$
\liminf _{n \rightarrow \infty} \sum_{i=n_{0}}^{n} \sum_{j=n_{0}}^{n} \mathbb{P}\left(A_{i} \cap A_{j}\right) /\left(\sum_{i=n_{0}}^{n} \mathbb{P}\left(A_{i}\right)\right)^{2} \leqslant C,
$$


which, by means of Kochen and Stone's (1964) Borel-Cantelli lemma, tells us that $\mathbb{P}\left(A_{n}\right.$; i.o. $) \geqslant 1 / C$. The proof of the divergent part of Theorem 1.2 is completed by a $0-1$ law similar to Lemma 2.3 .

The divergent half of Theorem 1.1 can be proved using the Borel-Cantelli lemma, by considering the events $A_{n}=\left\{V_{1}\left(\tau\left(t_{n}\right)\right) / \tau\left(t_{n}\right)>1-1 / \hat{\phi}\left(t_{n}\right)\right\}$, where $\tau$ is the inverse of the local time at 0 introduced in Section 1 , and $\hat{\phi}(t)=\phi\left(t^{3}\right)$. Moreover, it proves the first result in Theorem 5.1 (cf. Section 5). However, it is more convenient to apply a known integral test for the last passage process.

Fact 4.1 (Chung and Erdös 1952; Hobson 1992; 1994; Bertoin 1995). Let $\Lambda^{-}(t)$, be, as before, the last zero of $R$ before $t$. For any non-decreasing function $f>0$,

$$
\mathbb{P}\left[f\left(\Lambda^{-}(t)\right)<t \text {; i.o. }\right]=\left\{\begin{array} { l } 
{ 0 } \\
{ 1 }
\end{array} \Leftrightarrow \int ^ { \infty } \frac { \mathrm { d } t } { t ^ { 1 - | v | } ( f ( t ) ) ^ { | v | } } \left\{\begin{array}{c}
<\infty \\
=\infty
\end{array} .\right.\right.
$$

In the case $d=1$, the above test was established by Chung and Erdös (1952) and Hobson (1994). It was extended to Bessel processes by Hobson (1992) and to general Markov processes by Bertoin (1995).

Proof of the divergent half of Theorem 1.1. Let $\phi$ be a non-decreasing function such that $\int^{\infty}(\mathrm{d} t / t) \phi(t)^{-|v|}=\infty$. Let $f(t)=t \phi\left(t^{2}\right)$, which is non-decreasing. It is easily seen that $\int^{\infty} t^{-(1-|v|)}(f(t))^{-|v|} \mathrm{d} t=\infty$. Therefore, by the test in Fact 4.1, $\mathbb{P}\left(f\left(\Lambda^{-}(t)\right)<t\right.$; i.o. $)=1$. Since by the same test, $\Lambda^{-}(t) \geqslant t^{1 / 2}$ for large $t$, this yields $\mathbb{P}\left(\Lambda^{-}(t) \phi(t)<t\right.$; i.o. $)=1$. The divergent half of Theorem 1.1 follows using the obvious relation $\sum_{1}^{k} V_{j}(t) \geqslant t-\Lambda^{-}(t)$.

Proof of the divergent half of Theorem 1.4. If $\int{ }^{\infty}(\mathrm{d} t / t) \psi(t)^{-|v|}=\infty$, then by Theorem 1.1, $\mathbb{P}\left(V_{1}(t)>t(1-1 / \psi(t))\right.$; i.o. $)=1$. Since for any $k \geqslant 2, V_{k}(t) \leqslant t-V_{1}(t)$, this yields the desired conclusion.

Remark. J. Bertoin kindly points out that Theorem 1.1 can also be obtained by means of subordinator techniques. The present proof is retained because it gives a unified approach for this kind of problem.

\section{Excursion lengths à la Knight}

In the previous sections, we have studied the ordered lengths of Bessel excursions before $t$, by considering $\left(\Lambda^{-}(t), t\right)$ as an excursion interval. It looks natural to investigate also the completed excursion intervals, i.e. those before time $\Lambda^{-}(t)$. Knight (1986) established some interesting exact results for the longest completed excursion length of a Wiener process. Extensions to Bessel processes can be found in Perman (1993). Let

$$
\tilde{V}_{1}(t) \geqslant \tilde{V}_{2}(t) \geqslant \ldots \geqslant \tilde{V}_{n}(t) \geqslant \ldots
$$


be the ordered lengths of completed excursions of $R$ over $(0, t)$. One may ask whether the $\tilde{V}_{k}$ have the same asymptotic behaviours as the $V_{k}$.

Theorem 5.1. Let $k \geqslant 1$ and let $\phi>0$ be a non-decreasing function. Then

$$
\begin{array}{r}
\mathbb{P}\left[\sum_{j=1}^{k} \tilde{V}_{j}(t)>t\left(1-\frac{1}{\phi(t)}\right) ; \text { i.o. }\right]=\left\{\begin{array} { l } 
{ 0 } \\
{ 1 }
\end{array} \Leftrightarrow \int ^ { \infty } \frac { \mathrm { d } t } { t ( \phi ( t ) ) ^ { | v | } } \left\{\begin{array}{l}
<\infty \\
=\infty
\end{array},\right.\right. \\
\mathbb{P}\left[\sum_{j=1}^{k} \tilde{V}_{j}(t)<\frac{t}{\phi(t)} ; \text { i.o. }\right]=\left\{\begin{array} { l } 
{ 0 } \\
{ 1 }
\end{array} \Leftrightarrow \int ^ { \infty } \frac { \mathrm { d } t } { t ( \phi ( t ) ) ^ { | v | } } \left\{\begin{array}{l}
<\infty \\
=\infty
\end{array},\right.\right. \\
\mathbb{P}\left[\tilde{V}_{k}(t)<\frac{t}{\phi(t)} ; \text { i.o. }\right]=\left\{\begin{array} { l } 
{ 0 } \\
{ 1 }
\end{array} \Leftrightarrow \int ^ { \infty } \frac { \mathrm { d } t } { t ( \phi ( t ) ) ^ { | v | } } \left\{\begin{array}{l}
<\infty \\
=\infty
\end{array} .\right.\right.
\end{array}
$$

We feel free to omit the proof, which is more or less in the same vein as for the $V_{k}$. Comparing Theorem 5.1 with the corresponding results for the $V_{k}$, it is immediately seen that $\sum_{j=1}^{k} \tilde{V}_{j}(t)\left(\tilde{V}_{l}(t), l \geqslant 2\right)$ has the same upper (lower) functions as $\sum_{j=1}^{k} V_{j}(t)\left(V_{l}(t)\right.$, $l \geqslant 2)$. On the other hand, $\sum_{j=1}^{k} \tilde{V}_{j}(t)$ has completely different lower functions from $\sum_{j=1}^{k} V_{j}(t)$. This is due to the fact that the last zero-free interval $\left(\Lambda^{-}(t), t\right)$ has an overwhelming contribution in the liminf behaviour of $\sum_{j=1}^{k} V_{j}(t)$ (or simply in that of $\left.V_{1}(t)\right)$.

The problem of characterizing Lévy's upper class for $\tilde{V}_{k}(t)(k \geqslant 2)$ remains, to the best of our knowledge, open.

\section{Acknowledgements}

We are grateful to Catherine Rainer and Marc Yor for helpful discussions and references. Special thanks go to Jean Bertoin for insightful comments and for pointing out an elegant proof of Theorem 1.1 using subordinator techniques. We are also indebted to an anonymous referee for his careful reading and invaluable suggestions.

\section{References}

Bertoin, J. (1995) Sample path behaviour in connection with generalized arcsine laws. Probab. Theory Related Fields, 103, 317-327.

Chesney, M., Jeanblanc-Picqué, M. and Yor, M (1997) Brownian excursions and Parisian options. Adv. Appl. Probab., 29, 165-184.

Chung, K.L. and Erdös, P. (1952) On the application of the Borel-Cantelli lemma. Trans. Amer. Math. Soc., 72, 179-186.

Csáki, E., Erdös, P. and Révész, P. (1985) On the length of the longest excursion. $Z$. Wahrscheinlichkeitstheorie Verw. Geb., 68, 365-382. 
Erdös, P. (1942) On the law of the iterated logarithm. Ann. Math., 43, 419-436.

Feller, W. (1971) An Introduction to Probability Theory and Its Applications, Vol. II (2nd edn). New York: Wiley.

Hobson, D.G. (1992) Topics in the long-time behaviour of diffusions. PhD thesis, University of Cambridge.

Hobson, D.G. (1994) Asymptotics for an arcsin type result. Ann. Inst. H. Poincaré Probab. Statist., 30, $235-243$.

Jeulin, T. (1980) Semi-martingales et Grossissement d'une Filtration, Lecture Notes in Math. 833. Berlin: Springer-Verlag.

Knight, F.B. (1986) On the duration of the longest excursion. In E. Çinlar, K.L. Chung and M.J. Sharpe (eds), Seminar on Stochastic Processes 1985, Progr. Probab. Statist. 12, pp. 117-147. Boston: Birkhäuser.

Kochen, S.B. and Stone, C.J. (1964) A note on the Borel-Cantelli lemma. Illinois J. Math., 8, 248251.

Molchanov, S.A. and Ostrovski, E. (1969) Symmetric stable processes as traces of degenerate diffusions. Theory Probab. Appl., 14, 128-131.

Perman, M. (1993) Order statistics for jumps of normalised subordinators. Stoch. Process. Appl., 46, 267-281.

Perman, M., Pitman, J.W. and Yor, M. (1992) Size-biased sampling of Poisson point processes and excursions. Probab. Theory Related Fields, 92, 21-39.

Pitman, J.W. and Yor, M. (1992) Arcsine laws and interval partitions derived from a stable subordinator. Proc. London Math. Soc. (3), 65, 326-356.

Pitman, J.W. and Yor, M. (1997) The two-parameter Poisson-Dirichlet distribution derived from a stable subordinator. Ann. Probab. To appear.

Revuz, D. and Yor, M. (1994) Continuous Martingales and Brownian Motion (2nd edn). Berlin: Springer-Verlag.

Tenenbaum, G. (1995) Introduction to Analytic and Probabilistic Number Theory. Cambridge: Cambridge University Press.

Yor, M. (1995) Local Times and Excursions for Brownian Motion: A Concise Introduction. Lecciones Mat., Universidad Central de Venezuela.

Received February 1996 and revised July 1996. 